\title{
Variation in sensitivity and rate of change in body composition: steps toward individualizing transgender care
}

\author{
Daan M van Velzen ${ }^{(1}$, Nienke M Nota', Suat Simsek ${ }^{1,2}$, Elfi B Conemans ${ }^{1}$, \\ Guy T'Sjoen ${ }^{3,4}$ and Martin den Heijer' ${ }^{1}$
}

'Division of Endocrinology, Department of Internal Medicine, Amsterdam University Medical Center, Amsterdam, The Netherlands, ${ }^{2}$ Northwest Clinics, Department of Endocrinology, Alkmaar, The Netherlands, ${ }^{3}$ Department of Endocrinology, and ${ }^{4}$ Center for Sexology and Gender, Ghent University Hospital, Ghent, Belgium

Correspondence should be addressed to $\mathrm{M}$ den Heijer

Email

m.denheijer@vumc.n!

\begin{abstract}
Objective: Transgender individuals sometimes report a lack of physical change during hormone treatment, such as alterations in muscle tone or fat distribution. Identifying characteristics of this subgroup could be a step toward individualizing hormone therapy in transgender individuals. Therefore, we study the variation of changes in body composition and characteristics associated with a lack of change.

Design and methods: Body composition measures were recorded in 323 transmen and 288 transwomen at every visit from the start of hormone therapy to a maximum of 24 months follow-up. Absence of change was defined as transmen with a decrease in lean body mass or transwomen with a decrease in fat percentage.

Results: A lack of change at 24 months was observed in 19 of 94 (20.2\%) transmen and in 9 of 96 (9.4\%) transwomen. The risk of not achieving change in body composition was related to lower testosterone levels and less suppression of LH in transmen (OR: $0.67,95 \% \mathrm{Cl}: 0.48-0.94$ per SD increase in testosterone and OR: 1.36, 95\% Cl: 1.01-1.83 per SD increase in $\mathrm{LH}$ ).

Conclusions: There is a large variation in body composition changes during hormone therapy, with a substantial proportion of individuals with no measurable effects. In transmen, serum testosterone and LH were associated with a lack of change, but serum hormone levels were not associated with body composition changes in transwomen. The results provide a rationale for individualizing hormone therapy in transmen, by considering individual effects rather than solely relying on a standardized dosage of hormone therapy.
\end{abstract}

\section{Introduction}

Transgender people often desire to achieve body changes that bring their physical appearance closer to their experienced gender. Through the initiation of hormone therapy, the concentration of the sex hormones estrogen or testosterone is raised to the levels of the experienced gender, with the hope and expectation that secondary sex characteristics will follow accordingly.

One important effect of hormone therapy is change in body composition to the direction of the experienced gender (1). Transmen (birth assigned female, male gender identity) often seek an increase in muscle tone, while transwomen (birth assigned male, female gender identity) often desire a more feminine fat distribution (e.g. increased fat in the hip region), which may be quantified by measurement of fat percentage and lean body mass (LBM). The level of fat percentage generally ranges between 10 and $20 \%$ in healthy adult men, whereas a range of $20-30 \%$ is more common in healthy adult women (2). 
Although many papers have reported mean effects of hormonal treatment on body composition, none of these papers report on the distribution and variability of hormonal effects $(3,4,5)$. Such information is relevant, as in clinical practice, doctors are confronted with patients who report no change at all, despite adequate hormone levels. Such an experience is disappointing for the transgender person, but also for the treating physician who might be wondering whether the dosage of hormone therapy is adequate for this specific individual. Dosing in hormone therapy is currently solely based on arbitrary broad reference ranges of serum hormone levels with a standardized hormone dose for all, while individual effects of therapy are not considered (1). Objectively measuring the effects of hormone therapy and studying the determinants of effect, may aid in tailoring hormone therapy to the individual.

To improve transgender care, it would benefit physicians to get insight in the different response to hormone therapy among different transgender individuals. Identifying clinical differences between 'good' and 'bad' responders will probably aid in guiding hormone therapy in transgender individuals.

Therefore, the aim of the current study is to describe the variability of change in body composition measures among a large group of transgender individuals and to identify characteristics that may explain the observed lack of change. To do so, we study the dynamics of body composition measures during hormone therapy in all participants of the European Network for the investigation of Gender Incongruence (ENIGI) study in the Netherlands and Belgium, where bioelectrical impedance analysis (BIA) measurements are routinely performed at every clinic visit.

\section{Methods}

\section{Study design}

The current study is embedded in the European Network for the Investigation of Gender Incongruence (ENIGI). ENIGI is a partnership of currently five gender identity clinics in Amsterdam (the Netherlands), Ghent (Belgium), Oslo (Norway), Florence (Italy) and Tel Aviv (Israel). ENIGI was initiated to obtain more insight in the potential diversity in diagnostics and treatment of transgender individuals. Starting from 2010, participants were included in the ENIGI endocrine study when they started gender-affirming hormone therapy. Subjects were eligible to participate if they had not used transgender hormone therapy before and if they had sufficient knowledge of the native languages. At the start of hormone therapy, subjects received oral and written information of the ENIGI endocrine protocol by their physician and informed consent was obtained according to the institutional guidelines. The study was approved by the local ethics committee (METc VUmc, Amsterdam). A full overview of the ENIGI endocrine protocol has been published previously (6).

In this study, all participants included in the ENIGI endocrine protocol from January 2016 to December 2019 in Amsterdam and Ghent were eligible for inclusion. Subjects with less than two clinic visits were excluded.

\section{Treatment protocol}

Transmen received either testosterone gel $(50 \mathrm{mg}$ once daily), intramuscular (IM) injection of testosterone ester mixture (250 mg once per 3 weeks) or IM injection of testosterone undecanoate (1000 mg once every 12 weeks). Transwomen were treated with oral or transdermal application of estradiol ( $2 \mathrm{mg}$ twice daily or $100 \mu \mathrm{g}$ patches every 3 days, respectively), usually in combination with the anti-androgen cyproterone acetate (25 or $50 \mathrm{mg}$ daily). During follow-up, the dosage of hormones therapy was adjusted in case of inadequate serum testosterone or estradiol levels, according to standard clinical practice (1). Participants were not eligible for gender-affirming genital surgery prior to completion of 12 months of hormone therapy.

\section{Data collection}

Bioelectrical impedance analyses were performed using the Tanita MC-780 MA (Tanita Europe B.V., Amsterdam, The Netherlands) in both study centers. The Tanita MC-780 MA device consists of eight electrodes, measuring impedance using multifrequency $(1,10,50,250,500,1000$ $\mathrm{kHz}$ ) BIA. Machine outputted values of fat percentage were recorded. LBM and fat percentage were expressed as a proportion to the total amount of body fat. Measurements were performed in light clothing and bare feet with the removal of any accessories with metal compounds. Body weight was measured to the nearest $0.1 \mathrm{~kg}$ using the integrated scale on the BIA device. Body height was measured to the nearest centimeter using a Harpenden stadiometer. BMI was reported as the quotient of total body weight in kilograms divided by height squared in centimeters. BIA measurements were routinely performed at each outpatient clinic visit. 
In Amsterdam, estradiol was measured using liquid chromatography tandem mass spectrometry (LC-MS/ MS) (AUMC, Amsterdam, the Netherlands) with an interassay CV of $7 \%$ and an LOQ of $20 \mathrm{pmol} / \mathrm{L}$. Testosterone and luteinizing hormone (LH) were measured using a competitive immunoassay (Architect; Abbott) with an inter-assay CV range of $6 \%$ to $10 \%$ and an LOQ of 0.1 $\mathrm{nmol} / \mathrm{L}$ for testosterone and an inter-assay CV of $4-6 \%$ and LOQ of 2 IU/L for LH. After October 12th 2018, testosterone was measured using LC-MS/MS (AUMC, Amsterdam, the Netherlands) with an inter-assay CV range of $4-9 \%$ and LOQ of $0.1 \mathrm{nmol} / \mathrm{L}$. Comparability between the measurement of testosterone by the competitive immunoassay and LC-MS/MS has been previously published (slope: 1.05, r: 0.97) $(7,8)$.

In Ghent, estradiol was measured using an electrochemiluminescence immunoassay (ECLIA) on a E170 Modular (Gen III; Roche Diagnostics), with an inter-assay $\mathrm{CV}$ of $3.2 \%$ and an LOQ of $25 \mathrm{pmol} / \mathrm{L}$. Measurement of estradiol by ECLIA was comparable to ID-GC/MS (slope $1.00, \mathrm{r} 0.99)$. ECLIA E170 Modular was also used to measure testosterone and $\mathrm{LH}$ in Ghent. For testosterone, the inter-assay CV was $5 \%$ and LOQ $0.4 \mathrm{nmol} / \mathrm{L}$. For LH, the inter-assay CV was $6.4 \%$ and LOQ $1 \mathrm{IU} / \mathrm{L}$. Comparability between ECLIA and LC-MS/MS with regard to testosterone measurement has been previously published (slope 0.88 , r 0.98) (9).

BIA data and laboratory data were collected at the start of hormone therapy and during subsequent visits after 3, 6, 12, 18 and 24 months. Testosterone, estradiol and LH were determined in both transmen and transwomen.

\section{Statistical analysis}

Variables are displayed as mean $( \pm$ S.D. $)$ or median (interquartile range) in case of normal or non-normal distributed data, respectively. Ridgeplots (multiple density plots over time) were created to depict the distribution of fat percentage and LBM over time (e.g. 0, 3, 6, 12, 18 and 24 months follow-up) and to portray the individual change in fat percentage and LBM over time compared to the baseline value. A linear mixed model with a random intercept for subject, random slope for time and an unstructured covariance matrix was used to study mean changes over time.

Logistic mixed models with a random intercept for subject and study center were used to identify characteristics associated with a lack of change in body composition measures. A dichotomous variable was created, indicating whether a change or no change in body composition had occurred. A lack of increase in LBM was considered the outcome variable for transmen and a lack of increase in fat percentage was entered as the outcome variable for transwomen. A ratio value smaller than 1 of the outcome variables at a certain timepoint compared to the baseline value was recorded as no change (e.g. a fat percentage level of $8 \%$ at 12 months compared to a level of $12 \%$ at baseline would result in a ratio of 0.67 and thus be recorded as no change in transwomen, where an increase in fat percentage is expected).

Age at start of hormone therapy, baseline BMI, duration of hormone therapy (expressed at number of clinic visits) and mean hormone levels (estradiol, testosterone and LH) were considered potential determinants and were entered as dependent variables. A final adjusted model was created using backward selection.

A separate analysis was performed to determine if the amount of change after three months of hormone therapy is an indicator change at later time points. To do this, change over time was plotted and stratified by four groups, which represent different quartiles of the amount of change after three months.

Baseline values (prior to the start of hormone therapy) were excluded from the mean hormone level calculations. Testosterone levels of transmen using testosterone ester mixture were excluded from the multivariate analyses using mean serum levels of testosterone as a covariate, due to the strongly fluctuating levels of serum testosterone in this specific group. As measurements were performed during scheduled clinic visits, blood withdrawal did not coincide with a fixed dosage interval (e.g. trough levels). Therefore, mean serum testosterone values in users of testosterone ester mixture yield unreliable information. A sensitivity analysis was performed, including testosterone levels of transmen using testosterone ester mixture, to assess whether this influenced the final results.

Stata statistical software version 15.1 (StataCorp) was used for statistical analyses and graphics were created using the $\mathrm{R}$ version 3.6.3 software package (R Core Team, Vienna, Austria).

\section{Results}

\section{Variability of the change in body composition}

The total study population consisted of 323 transmen and 288 transwomen. The mean number of study visits was $4.0( \pm 1.0)$ for both transmen and transwomen. The characteristics of the study population are displayed in Table 1. 
Fat and LBM percentage moved in opposite directions in transmen and transwomen. After 24 months, a relative increase in LBM of 5.9\% (95\% CI 4.7-7.1) was observed in transmen with a range between $-17 \%$ and $+32 \%$. In transwomen, a relative increase in fat percentage of $30.0 \%$ (95\% CI 26.5-33.4) was observed, ranging between $-21 \%$ and $+190 \%$. Figure 1 displays the population distribution of fat percentage and LBM values over time. Figure 2 displays the population distribution of the relative change in fat percentage and LBM compared to baseline.

\section{Lack of change}

As described earlier, a negative or zero percent change in LBM or fat percentage was defined as no change in transmen and transwomen, respectively. Of all study visits, no change was present in 395 of 1658 observations
(23.8\% overall, $28.0 \%$ in transmen and $19.2 \%$ in transwomen). After 12 months of hormone therapy, no change was observed in 65 of 242 transmen (26.9\%) and 38 of 229 transwomen (16.6\%). Of those that underwent a clinical visit at 24 months, the prevalence of no change decreased to $20.2 \%(n=19$ of 94$)$ in transmen and $9.4 \%$ $(n=9$ of 96$)$ in transwomen.

\section{Determinants of lack of change in body composition}

In transmen, both low mean testosterone levels and high mean LH levels were associated with no change. A 1 s.D. increase in testosterone (s.D.: $10.9 \mathrm{nmol} / \mathrm{L}$ ) was associated with lower odds of no change (OR: 0.67, 95\% CI: 0.48-0.94) and a 1 s.D. increase in LH (s.D.: 4.5 IU/L) with higher odds of no change (OR: 1.36, 95\% CI:

Table 1 Population characteristics.

\begin{tabular}{l}
\hline \\
\hline Baseline characteristics \\
Study center, $n$ (Amsterdam/Ghent) \\
Number of study visits \\
Age \\
Weight $(\mathrm{kg})$ \\
Height $(\mathrm{cm})$ \\
BMI $\left(\mathrm{kg} / \mathrm{m}^{2}\right.$ ) \\
Total body fat $(\mathrm{kg})$ \\
Fat percentage $(\%)$ \\
Lean body mass $(\mathrm{kg})$ \\
Lean body mass percentage \\
Last used type of hormonal medication, $n$ \\
Testosterone gel \\
Testosterone ester mixture \\
Testosterone undecanoate \\
Estradiol tablets \\
Estradiol patches \\
Anti-androgen treatment, \\
CPA \\
Other \\
None \\
Daily CPA dose $(n)$ (mg) \\
10 \\
25 \\
50
\end{tabular}

\begin{tabular}{|c|c|}
\hline Transmen $(n=323)$ & Transwomen $(n=288)$ \\
\hline $280 / 43$ & $263 / 25$ \\
\hline $4.0 \pm 1.0$ & $4.0 \pm 1.0$ \\
\hline $21(19-25)$ & $25(21-38)$ \\
\hline 67.1 (58.9-79.6) & $71.9(62.6-82.9)$ \\
\hline $168 \pm 7$ & $179 \pm 7$ \\
\hline $23.2(20.7-28.3)$ & $22.3(19.7-24.6)$ \\
\hline $20.6(14.1-31.8)$ & $12.1(7.9-16.8)$ \\
\hline $31.3(23.8-40.3)$ & $16.6(12.6-21.3)$ \\
\hline 45.7 (42.4-49.9) & 59.7 (54.4-66.0) \\
\hline 68.7 (59.7-76.2) & 83.3 (78.7-87.4) \\
\hline 148 & N/A \\
\hline 77 & N/A \\
\hline 98 & N/A \\
\hline N/A & 174 \\
\hline N/A & 114 \\
\hline \multirow[t]{4}{*}{ N/A } & \\
\hline & 276 \\
\hline & 10 \\
\hline & 2 \\
\hline \multirow[t]{4}{*}{ N/A } & \\
\hline & 9 \\
\hline & 217 \\
\hline & 50 \\
\hline $168(85-368)$ & $79(65-96)$ \\
\hline $1.1(0.8-1.4)$ & $18(13-23)$ \\
\hline $3.7(2.2-6.4)$ & $3.3(2.3-4.7)$ \\
\hline 144 (108-199) & $230(169-326)$ \\
\hline $18.2(14.0-24.5)$ & $0.5(0.4-0.8)$ \\
\hline $3.8(2-6.2)$ & $0.1(0.1-0.5)$ \\
\hline
\end{tabular}

*Testosterone ester mixture users excluded due to fluctuating serum testosterone levels; ${ }^{\dagger} \mathrm{A}$ single individual used a GnRH-analog and another used spironolactone as an anti-androgen agent.

CPA, cyproterone acetate; $\mathrm{HT}$, hormone therapy; $\mathrm{LH}$, luteinizing hormone. 


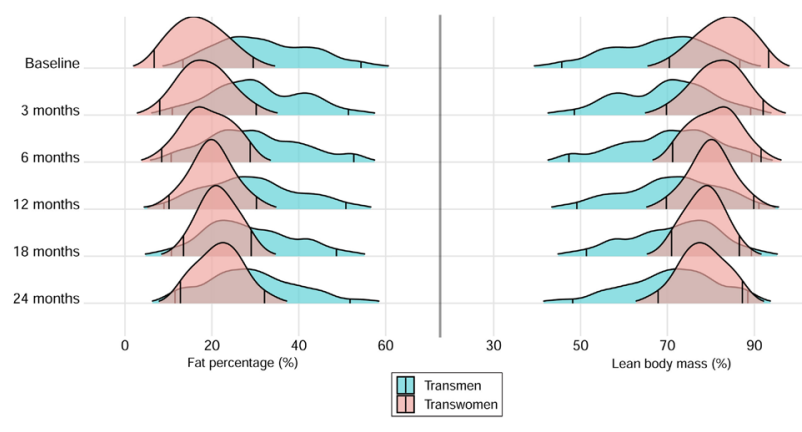

Figure 1

Distribution of fat percentage and lean body mass during hormone therapy. Both fat percentage and lean body mass are expressed as a fraction of total body weight. The $y$-axis represents the density of probability for a given value of fat percentage or lean body mass.

1.01-1.83). In transwomen, no associations were found between serum hormone levels and lack of change in fat percentage.

In both transmen and transwomen, age at the start of HT was not associated with a lack of change in body composition measures (OR: 0.99, 95\% CI: 0.96-1.02 and OR: 1.01, 95\% CI: 0.99-1.02 for transmen and transwomen, respectively). The amount of change at three months was divided into quartiles to assess whether these results predicted change in body composition at later time points. The results are plotted in Fig. 3, displaying the mean changes in body composition over time, stratified by quartiles of change after three months of hormone therapy. Figure 3 shows that individuals with a lack of change after 3 months of hormone therapy remained in the lowest quartile of change during the remainder of the study follow-up. Table 2 shows the results of

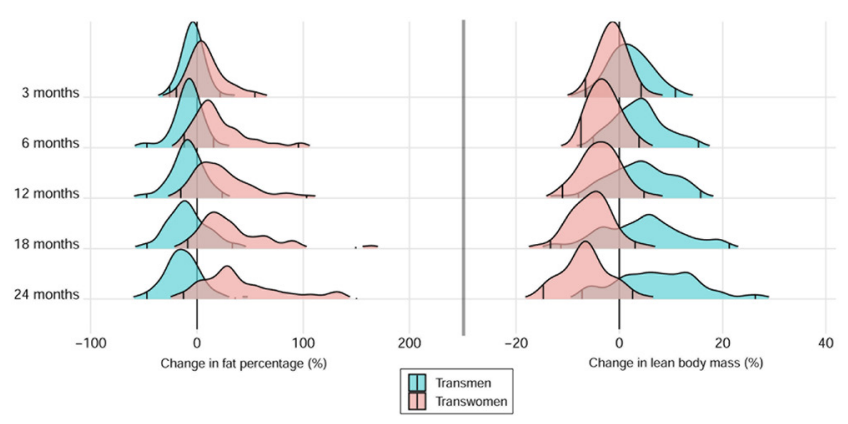

\section{Figure 2}

Change in fat percentage and lean body mass during hormone therapy compared to baseline. The $y$-axis represents the density of probability for a given value of fat percentage or lean body mass.
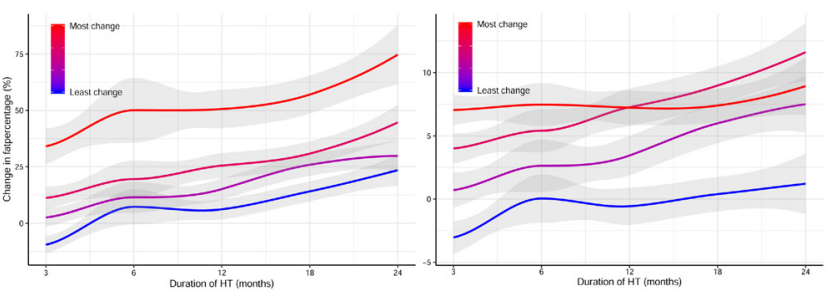

\section{Figure 3}

Mean relative changes in fat percentage and lean body mass, stratified by the amount of change after 3 months of hormone therapy. Left, transwomen and right, transmen. Shaded area represents $\pm 95 \% \mathrm{Cl}$. LBM, lean body mass;

$\mathrm{HT}$, hormone therapy.

logistic mixed model analyses evaluating all proposed determinants of no change in body composition measures. All reported associations remained present in a backwards selected model (final model OR's presented above). A sensitivity analysis, including serum testosterone levels from transmen using testosterone ester mixture did not yield different results (data not shown).

\section{Discussion}

Previous literature on hormone therapy in transgender individuals has had a main focus on mean or group effects.

The observation that some transgender people report no effects of hormone therapy has shifted our attention to study the between-subject variability of hormone effects and explore the possibility of a personalized approach to hormone therapy, rather than a 'one size fits all' treatment regime. The current study has focused on changes in body composition as a measure for hormone effects, a key feature of this hormone therapy.

In 323 transmen and 288 transwomen, the mean relative changes in body fat and LBM during hormone therapy were comparable to previous studies in literature $(3,4,5,10,11)$. While most of the aforementioned studies have reported only data from two different time points (e.g. changes from baseline to 12 months follow up), we add by showing that the relative changes in body fat seem to follow a linear pattern over multiple measurements in time. A single study group has reported changes in body composition beyond one year of hormone therapy, and reported ongoing changes in body composition in transwomen, but not in transmen $(10,11)$. In concurrence, we found ongoing changes in body composition in transwomen, but also in transmen. This may be explained by differences in the definition of LBM between studies. 
Table 2 Determinants of lack of change in body composition. Data are presented as OR $(95 \% \mathrm{Cl})$.

Age at start of HT (years)
Duration of HT (per number of
$\quad$ clinic visits)
BMI at start of HT
$\quad$ (per 5 units increase)
Serum testosterone
$\quad$ (per SD increase)
Serum estradiol
$\quad$ (per SD increase)
LH (per SD increase)

\begin{tabular}{c}
\hline Unadju \\
\hline $0.99(0.96-1.02)$ \\
$\mathbf{0 . 5 6}(\mathbf{0 . 4 9 - 0 . 6 4 )}$ \\
$\mathbf{0 . 7 1}(\mathbf{0 . 5 7 - 0 . 8 8 )}$ \\
$\mathbf{0 . 7 0}(\mathbf{0 . 5 1}-\mathbf{0 . 9 6 )}$ \\
$0.96(0.73-1.26)$ \\
$\mathbf{1 . 3 6}(\mathbf{1 . 0 6 - 1 . 7 4 )}$
\end{tabular}

Unadjusted

Transwomen

$1.01(0.99-1.02)$

$0.49(0.41-0.58)$

$1.63(1.31-2.03)$

$1.08(0.86-1.37)$

$0.97(0.76-1.25)$

$1.08(0.86-1.36)$

\begin{tabular}{ccc}
\hline \multicolumn{2}{c}{ Adjusted* } \\
\hline Transmen & & Transwomen \\
$0.60(0.51-0.70)$ & & $0.48(0.41-0.57)$ \\
$0.72(0.54-0.97)$ & & $1.76(1.36-2.29)$ \\
$0.67(0.48-0.94)$ & $N / A$ \\
$N / A$ & $N / A$ \\
$1.36(1.01-1.83)$ & N/A \\
\hline
\end{tabular}

*BMI at start of HT, duration of HT, serum testosterone and serum LH were included in the final model for transmen. Adjusted model for transwomen included BMI at start of HT and duration of HT.

S.D. for transmen: testosterone $10.9 \mathrm{nmol} / \mathrm{L}$, estradiol $105 \mathrm{pmol} / \mathrm{L}, \mathrm{LH}: 4.5 \mathrm{IU} / \mathrm{L}$. S.D. for transwomen: testosterone $3.1 \mathrm{nmol} / \mathrm{L}$, estradiol $149 \mathrm{pmol} / \mathrm{L}$, LH: 1.8 IUI/L. HT, hormone therapy; LH, luteinizing hormone.

In the current study, LBM was presented as a proportion of total body weight, rather than an absolute measure in kilograms. A concomitant decrease in fat mass in transmen thus affects LBM when using the first, but not the second definition.

However, aside from mean changes, a large distribution or range in body composition change was observed in both transmen and transwomen, which tended to increase over time. This observation highlights that over time, mean population changes are less applicable to the individual and that the variability of hormonal effects increases with the duration of hormone therapy. The latter observation is understandable, as the effects of hormone therapy may be complemented or negated by other variables (e.g. physical activity or dietary habits), of which the effects usually manifest over time.

Of particular interest was the proportion of observations without a change in body composition. Of all individual observations, this proportion amounted to a total of $23.8 \%$. The number of individuals characterized as no change (e.g. no increase in fat percentage in transwomen, or no increase in LBM in transmen) decreased with the duration of hormone therapy, but was still present in as much as $20.2 \%$ of transmen and $9.4 \%$ of transwomen after 24 months of hormone therapy. By comparing the characteristics of these people with the remainder of the population, we aimed to identify factors associated with a lack of change in body composition.

First and most important, the association of a lack of increase in LBM with lower testosterone levels and higher LH levels suggests insufficiently dosed hormone therapy may often be the cause of a lack of change in transmen. This dose-response relationship was not present in transwomen, in which neither estradiol or testosterone levels were associated with a lack of change in body composition. Interestingly, this may highlight that the physical effects of hormone therapy are not linearly related to the serum estradiol level in transwomen. Body composition change may therefore only be a suitable surrogate marker for hormone therapy in transmen, but not in transwomen. However, more detailed information on estrogens in transwomen may give additional insights regarding the effects of other sex steroids on body composition change. Estrone (E1) and the ratio of estradiol (E2) to E1, in particular, are a subject of interest for future studies. In addition, determination of sex hormone-binding globulin would have yielded additional information regarding the bioavailability of sex steroids. Although not reported in the current study, it is additionally important to note that different formulations of testosterone and estradiol had identical effects on changes in body composition in a previous report from our study group (4).

Second, in transwomen and particularly in transmen, the amount of change in body composition which, had occurred after 3 months of hormone therapy was predictive of the response at future outpatient visits. While the overall odds of no change do decrease over time, Fig. 3 shows that, on average, individuals with the least effect after 3 months do not make up for this lack of change as the duration of hormone therapy progresses.

Third, although many transgender persons are afraid of experiencing fewer effects when they start hormone therapy at an older age, our study did not find a relationship between age and effect of hormone therapy on body composition. While age may possibly affect other end points of feminizing or masculinizing hormone therapy, this does not seem to be true for changes in body composition. This finding is in line with a previous report 
on body composition change and a previous study on breast growth in transwomen $(4,12)$.

Further, transmen without change were characterized by an on average lower BMI at baseline, whereas the opposite was true for transwomen. This might indicate that the lipolyzing effect of testosterone is less effective in those with a low amount of fat, and the lipogenic effects of estradiol are less effective in those with a surplus of body fat. However, the strong association with baseline BMI may also be partially explained by confounding. In our study center, a BMI smaller than $30 \mathrm{~kg} / \mathrm{m}^{2}$ is a prerequisite for genital surgery eligibility (e.g. vaginoplasty or phalloplasty). With this in mind, it is possible that the association between a higher BMI and a lack of change (a decrease in fat percentage) in transwomen is explained by a focus on weight loss in these individuals, rather than smaller hormonal effects. Similarly, BMI was higher in the group of transmen with an increase in LBM, which may also be the partial result of factors, such as diet and physical exercise.

These results may have the following implications for clinical practice. First, the effects of hormone therapy on body composition in individuals may largely differ from the reported mean effects. As the large range of effect is not entirely explained by individual (measurable) characteristics, it is important to emphasize that changes in body composition are not wholly predictable and are not guaranteed. In order to manage expectations, it may be valuable to discuss this topic with individuals at the start of hormone therapy.

Second, but most important, the association of body composition changes with testosterone and LH levels in transmen (prescribed either testosterone gel or undecanoate) may provide a rationale for using body composition measurements as a clinical tool for guiding the dosing of hormone therapy in transmen. This approach would incorporate individual effects of hormone therapy into clinical decision making, rather than utilizing a standard treatment for all, as is currently suggested in guidelines (1). For example, a higher testosterone dose may be given to individuals with low-normal testosterone levels and/or higher LH levels in combination with a recorded lack of effect, despite serum testosterone levels remaining within the appointed reference range. Change in body composition may also be especially useful for guiding hormone therapy in transmen using testosterone formulations that give fluctuating serum hormone levels, in whom random testosterone measurements thus yield little information. Finally, as a lack of change after 3 months of hormone therapy was predictive of the response at later outpatient visits, a higher dose of testosterone in this subgroup may be more easily considered.

While a more personalized approach to transgender hormone therapy is indeed already applied to some extent, it has not been previously supported by clinical research and is often based on subjective experiences. A viable method for objectively recording hormone effects has thus far been lacking. Based on these study results and considering the low-cost and practicality of BIA, it may be a useful tool to incorporate into clinical practice. The current study has focused solely on body composition as a measure of the effect of HT, as it illustrates and supports the concept of steering away from protocolized transgender endocrine care. However, it would be an interesting and important topic for future research to consider an identical study incorporating other end points and effects of HT such as bone health, suppression of menses or (facial) hair growth to identify 'non-responders'.

The current study is the largest longitudinal study on body composition changes in transgender individuals during gender-affirming hormone therapy and the first to focus on the variability of hormonal effects between individuals. In particular, this study is the first to discuss an individualized approach to hormone therapy in transmen. The absence of strict inclusion criteria in the ENIGI cohort makes the results fairly generalizable to the entire population of transgender individuals. Due to the low-cost and practicality of BIA measurements, we were able to perform multiple repeated measurements, which would not have been possible using more expensive and labor-intensive methods, such as DXA measurements. The study is also subject to a few limitations. First, although BIA measurements correspond highly to DXA measurements in healthy adults (13), the results are not validated in transgender individuals and BIA measurements in obesity must be interpreted with caution. Nevertheless, our study results are comparable to previous studies using DXA to measure body composition $(3,4,5)$, of which one was performed in the current study population (4), suggesting that BIA provides reliable estimates of fat percentage and LBM. Second, we used changes in total body fat as a marker for changes in body composition. However, with these data, we cannot draw conclusions on changes in regional body fat and body fat distribution. It may be possible that a large shift in body fat distribution (e.g. from waist to hip region) is observed, while the total body fat percentage remains equal. Finally, we were unable to control for confounding by lifestyle factors such as physical exercise and diet, which may have partly explained the variability in hormonal 
effects between individuals. This may be especially important, as the prevalence rates of eating disorders in transgender persons are higher compared to the general population (14).

In conclusion, even after two years of hormone therapy, body composition changes are still progressing. However, there is a large inter-individual variation, with a total of $20 \%$ of transmen and $9 \%$ of transwomen without measurable effects even after two years. The study results may aid in clinical counseling and supports a rationale for individualizing hormone therapy, based on our findings especially in transmen, by focusing on individual measures of effects, rather than following a standardized treatment for all. Further studies on variation in effects are indicated to individualize endocrine care of transgender persons.

\section{Declaration of interest}

The authors declare that there is no conflict of interest that could be perceived as prejudicing the impartiality of this study.

\section{Funding}

This research did not receive any specific grant from any funding agency in the public, commercial or not-for-profit sector.

\section{Acknowledgements}

This study is based on data collected in the framework of the European Network for the Investigation of Gender Incongruence (ENIGI) study. The authors would like to thank J Defreyne and S M Collet from the Ghent site for their contribution regarding the collection of data.

\section{References}

1 Hembree WC, Cohen-Kettenis PT, Gooren L, Hannema SE, Meyer WJ, Murad MH, Rosenthal SM, Safer JD, Tangpricha V \& T'Sjoen GG. Endocrine treatment of gender-dysphoric/gender-incongruent persons: an Endocrine Society clinical practice guideline. Journal of Clinical Endocrinology and Metabolism 2017102 3869-3903. (https:// doi.org/10.1210/jc.2017-01658)

2 Wells JC. Sexual dimorphism of body composition. Best Practice and Research: Clinical Endocrinology and Metabolism 200721 415-430. (https://doi.org/10.1016/j.beem.2007.04.007)

3 Van Caenegem E, Wierckx K, Taes Y, Schreiner T, Vandewalle S, Toye K, Lapauw B, Kaufman JM \& T'Sjoen G. Body composition, bone turnover, and bone mass in trans men during testosterone treatment: 1-year follow-up data from a prospective case-controlled study (ENIGI). European Journal of Endocrinology 2015172 163-171. (https://doi.org/10.1530/EJE-14-0586)
4 Klaver M, de Blok CJM, Wiepjes CM, Nota NM, Dekker MJHJ, de Mutsert R, Schreiner T, Fisher AD, T'Sjoen G \& den Heijer M. Changes in regional body fat, lean body mass and body shape in trans persons using cross-sex hormonal therapy: results from a multicenter prospective study. European Journal of Endocrinology 2018 178 163-171. (https://doi.org/10.1530/EJE-17-0496)

5 Fighera TM, da Silva E, Lindenau JD \& Spritzer PM. Impact of cross-sex hormone therapy on bone mineral density and body composition in transwomen. Clinical Endocrinology 201888 856-862. (https://doi.org/10.1111/cen.13607)

6 Dekker MJ, Wierckx K, Van Caenegem E, Klaver M, Kreukels BP, Elaut E, Fisher AD, van Trotsenburg MA, Schreiner T, den Heijer M et al. A European Network for the Investigation of Gender Incongruence: endocrine part. Journal of Sexual Medicine 201613 994-999. (https://doi.org/10.1016/j.jsxm.2016.03.371)

7 Bui HN, Sluss PM, Blincko S, Knol DL, Blankenstein MA \& Heijboer AC. Dynamics of serum testosterone during the menstrual cycle evaluated by daily measurements with an ID-LC-MS/MS method and a 2nd generation automated immunoassay. Steroids 2013 78 96-101. (https://doi.org/10.1016/j.steroids.2012.10.010)

8 Groenestege WM, Bui HN, ten Kate J, Menheere PP, Oosterhuis WP, Vader HL, Heijboer AC \& Janssen MJ. Accuracy of first and second generation testosterone assays and improvement through sample extraction. Clinical Chemistry 201258 1154-1156. (https://doi. org/10.1373/clinchem.2011.181735)

9 Chen Y, Yazdanpanah M, Hoffman BR, Diamandis EP \& Wong PY. Rapid determination of serum testosterone by liquid chromatography-isotope dilution tandem mass spectrometry and a split sample comparison with three automated immunoassays. Clinical Biochemistry 200942 484-490. (https://doi.org/10.1016/j. clinbiochem.2008.11.009)

10 Mueller A, Haeberle L, Zollver H, Claassen T, Kronawitter D, Oppelt PG, Cupisti S, Beckmann MW \& Dittrich R. Effects of intramuscular testosterone undecanoate on body composition and bone mineral density in female-to-male transsexuals. Journal of Sexual Medicine 20107 3190-3198. (https://doi.org/10.1111/j.17436109.2010.01912.x)

11 Mueller A, Zollver H, Kronawitter D, Oppelt PG, Claassen T, Hoffmann I, Beckmann MW \& Dittrich R. Body composition and bone mineral density in male-to-female transsexuals during cross-sex hormone therapy using gonadotrophin-releasing hormone agonist. Experimental and Clinical Endocrinology and Diabetes 2011119 95-100. (https://doi.org/10.1055/s-0030-1255074)

12 de Blok CJM, Klaver M, Wiepjes CM, Nota NM, Heijboer AC, Fisher AD, Schreiner T, T'Sjoen G \& den Heijer M. Breast development in transwomen after 1 year of cross-sex hormone therapy: results of a prospective multicenter study. Journal of Clinical Endocrinology and Metabolism 2018103 532-538. (https://doi. org/10.1210/jc.2017-01927)

13 Leahy S, O'Neill C, Sohun R \& Jakeman P. A comparison of dual energy X-ray absorptiometry and bioelectrical impedance analysis to measure total and segmental body composition in healthy young adults. European Journal of Applied Physiology 2012112 589-595. (https://doi.org/10.1007/s00421-011-2010-4)

14 Diemer EW, Grant JD, Munn-Chernoff MA, Patterson DA \& Duncan AE. Gender identity, sexual orientation, and eatingrelated pathology in a national sample of college students. Journal of Adolescent Health 201557 144-149. (https://doi.org/10.1016/j. jadohealth.2015.03.003) 